\title{
Análisis de integridad estructural de tuberías de material compuesto para el transporte de hidrocarburos por elementos finitos
}

\section{Structural integrity analysis of composite pipes for the transport of crude oil by finite elements}

\author{
O.A. González-Estrada ${ }^{1}$, J. Leal-Enciso ${ }^{2}$, J.D. Reyes-Herrera ${ }^{3}$ \\ ${ }^{1}$ GIEMA, School of Mechanical Engineering, Universidad Industrial de Santander, Colombia. Email: agonzale@uis.edu.co \\ ${ }^{2}$ GIEMA, School of Mechanical Engineering, Universidad Industrial de Santander, Colombia. \\ Email: joaquin.leal@correo.uis.edu.co \\ ${ }^{3}$ GIEMA, School of Mechanical Engineering, Universidad Industrial de Santander, Colombia.
}

Recibido: abr 12, 2016. Aceptado: jun 20, 2016. Versión final: jun 28, 2016

\begin{abstract}
RESUMEN
En este trabajo se investiga la caracterización del comportamiento mecánico de la tubería compuesta Fiberspar por medio de análisis por elementos finitos (FEA), con el fin de estudiar la integridad estructural de tuberías compuestas para el transporte de petróleo en Colombia. Se realizaron ensayos de laboratorio de acuerdo con la norma ASTM D2290 con el objetivo de determinar la resistencia a la tensión y comparar los resultados con el modelo numérico. Se utilizaron los criterios de fallo de Tsai-Wu y Tsai-Hill para la tubería trabajando a diferentes presiones, incluyendo la presión operacional, determinando valores para el fallo e identificando las capas críticas.
\end{abstract}

Palabras clave: Tubería material compuesto, análisis de elementos finitos, simulación, criterios de falla, ASTM D2290.

\begin{abstract}
In this work, we performed a finite element analysis (FEA) to characterize the mechanical behavior of the composite pipe Fiberspar in order to investigate the structural integrity of composite pipes for crude oil transport in Colombia. Tests according to ASTM D2290 standard were performed in the laboratory to determine the tensile ultimate strength and compare the results with the numerical model. Failure criteria Tsai-Wu and Tsai-Hill were considered for the pipes working at different pressures, including operational pressure and, thus, the failure values and critical layers were identified.
\end{abstract}

Keywords: Composite pipes, finite element analysis, simulation, failure criteria, ASTM D2290.

\section{INTRODUCCIÓN}

Durante los últimos años, diferentes tipos de tuberías de material compuesto se han empleado con éxito en los campos de petróleo y gas, debido a que sus propiedades mecánicas resultan muy atractivas, en especial su relación peso/resistencia y su resistencia a la corrosión [1]. Otras características como facilidad de instalación, alta durabilidad y facilidad de mantenimiento las hacen más deseables que las tuberías de acero. Diferentes estudios sobre las propiedades mecánicas y ensayos de laboratorio han sido llevados a cabo para su caracterización [2], [3].

De Sousa et al. [4] obtuvieron resultados de esfuerzos para la tubería compuesta tipo Riser, que contiene capas metálicas y termoplásticas, a partir de modelos teóricos y modelos numéricos. Anping et al. [5] realizaron un análisis de elementos finitos para determinar las propiedades mecánicas sobre dos tipos de tuberías compuestas reforzadas con alambres de acero.

Recientemente, Yu et al. [6] investigaron mediante elementos finitos el comportamiento a flexión de las tuberías compuestas termoplásticas RTP (reinforced thermoplastic pipes) reforzados con fibra de aramida. Los resultados relacionaron la falla por pandeo con los ángulos entre las capas de refuerzo. Yu et al. [7] realizaron modelos numéricos para la tubería RTP y concluyeron que la mejor resistencia al pandeo se obtiene cuando las fibras de la tubería están orientadas a $\left[ \pm 65^{\circ} \% \pm 75^{\circ}\right]$, también identificaron las mejores relaciones diámetro/espesor de la tubería. 
En Colombia se han realizado algunos estudios de las tuberías compuestas para el transporte de hidrocarburos mediante ensayos de laboratorio y así examinar el efecto de las ralladuras sobre la superficie de las tuberías [8].

Bai et al. [9] desarrollaron modelos numéricos para analizar el colapso de la tubería RTP, donde los resultados de la aproximación mediante elementos finitos (FEM) reflejan un porcentaje de error muy bajo con respecto al análisis teórico. Reutov [10] estudió mediante elementos finitos las tuberías multicapa "Soluforce" para aplicaciones de petróleo y gas, obteniendo los esfuerzos equivalentes para cada capa, donde se determinó que la capa media de refuerzo presenta los máximos esfuerzos para presiones de operación. En [11] se trabajó en una herramienta analítica para tuberías termoplásticas compuestas con el objetivo de correlacionar los resultados con ensayos de laboratorio como tensión, compresión, presión interna y externa.

En [12] se analizan tuberías compuestas de filamentos enrollables expuestas a cargas termomecánicas debidas al fluido caliente que circula dentro de ellas. En [13] los autores desarrollan modelos analíticos para dar una solución aproximada para tubos compuestos de filamento enrollado de varias capas bajo flexión.

Soden et al. [14] realizaron ensayos de rotura con muestras tubulares de laminados de fibra de vidrio $\mathrm{E} y$ epoxi, con $60 \%$ de resina y con ángulos de enrollamiento de $\pm 55^{\circ}$, obteniendo una tensión de rotura de $595 \mathrm{MPa}$. Las curvas de esfuerzo deformación mostraron comportamientos no lineales.

En este trabajo se realiza el análisis estructural de la tubería compuesta de fibra de vidrio Fiberspar, mediante el uso de elementos finitos, utilizando las condiciones de contorno dadas por la operación. Además, se comparan los resultados experimentales de acuerdo a la norma ASTM D2290 realizadas en laboratorio, con los resultados del modelo numérico. Se estudian los criterios de fallo de Tsai- Hill y Tsai-Wu para el análisis de la tubería a diferentes presiones, incluyendo la presión de operación.

\section{INTEGRIDAD ESTRUCTURAL DE LA TUBERÍA FIBERSPAR}

Las tuberías flexibles de material compuesto de tecnología de filamentos enrollables tienen un comportamiento anisótropo, debido a los diferentes ángulos que presentan las capas de refuerzo. Las tuberías pueden estar expuestas a cargas termomecánicas debido al fluido circulante, y se caracterizan por tener una baja rigidez a la flexión comparadas con tuberías de acero. La tubería Fiberspar está constituida por 3 capas principales [15]: capa interior y exterior de polietileno de alta densidad PE 3408 y un refuerzo de epoxi y fibra de vidrio tipo $\mathrm{E}$ (dividido en 2 subcapas a $55^{\circ}$, subcapas a $-55^{\circ}$ ) de refuerzo, las cuales están apiladas de manera simétrica, las fibras de cada refuerzo corresponden al orden de $[+55,-55,+55,-55]$. La Figura 1 ilustra la forma de las orientaciones de las fibras.

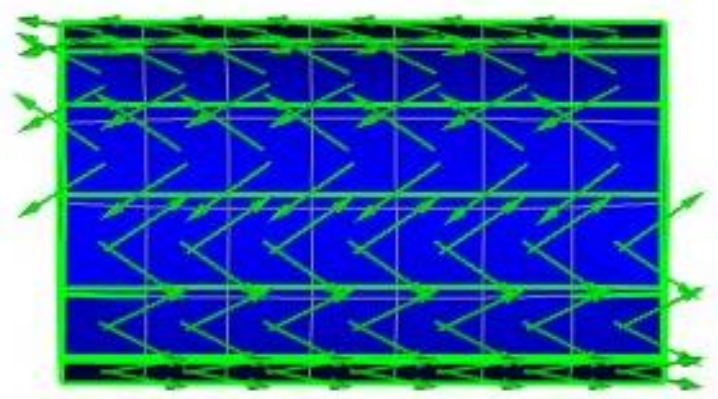

Figura 1. Orientación de las fibras de la capa de material compuesto. Fuente. Elaboración propia.

La Figura 2 ilustra la forma integral de la tubería, donde la primera capa de material corresponde a una capa termoplástica resistente al desgaste, la cual protege a la tubería de las condiciones externas a ellas. La segunda capa son fibras de vidrio reforzadas con epoxi, que permite mayor resistencia y flexibilidad de la tubería. La tercera capa es una capa de unión, la cual refuerza la fibra de vidrio y está unida con la cuarta capa, que funciona como una barrera de presión termoplástica y es la que entra en contacto con el fluido circulante.

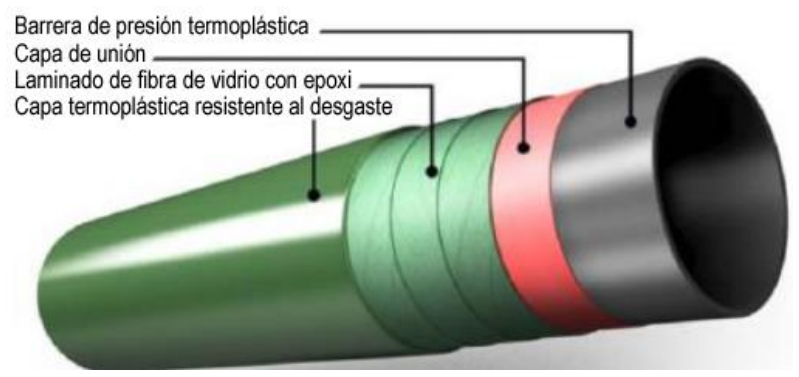

Figura 2. Tubería Fiberspar, modelo constructivo. Fuente: www.fiberflex.com.ex

\subsection{Condiciones comunes de operación}

La tubería Fiberspar en su catálogo presenta una variedad de condiciones de operación, las cuales oscilan entre 750 psi y 2500 psi, estas presiones se conocen como presión nominal o presión de funcionamiento. La tubería enrollable en el sector petrolero colombiano trabaja con una presión nominal de 750 psi $(5.17 \mathrm{MPa})$. Se han realizado pruebas de estallido para la tubería, las cuales oscilan entre 3940.6 psi $(27,17 \mathrm{MPa})$ y 4800 psi (31 $\mathrm{MPa}$ ). Esta prueba hace referencia a la máxima presión a la cual la tubería falla, también es conocida como prueba de explosión. 


\subsection{Esfuerzos en tuberías compuestas}

Kaynak et al. [16] realizaron ensayos de acuerdo a la norma ASTM D2290 para tuberías compuestas enrollables con fibras de vidrio o carbono unidas con resina epoxi, con el objetivo de estudiar el efecto de diferentes parámetros en el comportamiento mecánico. Se observó una fuerte dependencia de la resistencia a la tensión tangencial y el módulo de elasticidad con respecto al ángulo de enrollamiento (ver Figura 3). Con el ensayo ASTM D2290 se concluyó que las muestras de aro de fibra de vidrio con epoxi para ángulos de enrollamientos entre $\pm 45^{\circ} \mathrm{y} \pm 65^{\circ}$ presentan resistencia de tensión a la rotura entre $180 \mathrm{MPa}$ y $600 \mathrm{MPa}$, respectivamente.

Las tuberías de fibra de vidrio se consideran ortótropas por naturaleza. Las propiedades mecánicas en los tres ejes perpendiculares en los tubos GRP son distintos. Para las tuberías GRP de transporte de fluidos se prefiere que el ángulo ideal de bobinado o enrollamiento de la fibra sea de $\pm 55^{\circ}$ con respecto a la dirección axial.

Carroll et al. [17] realizaron un estudio experimental de tubos de fibra de vidrio y epoxi con ángulos de enrollamiento de $\pm 55^{\circ}$ con diámetro interno de 2 in. Se utilizó una máquina de ensayo que permite diferentes relaciones de cargas radial y axial. Las curvas de esfuerzo-deformación resultantes mostraron un comportamiento complejo de los tubos. La Figura 4 muestra los esfuerzos circunferenciales hasta la rotura cuando es aplicada una carga de presión pura (1 Circunferencial: 0 Axial) registrando esfuerzos máximos de $402 \mathrm{MPa}$ en dirección circunferencial.

El comportamiento en las tuberías de fibra de vidrio es elástico-lineal al inicio, seguido de un comportamiento no lineal cerca a la falla, primero por pequeñas fugas y, segundo, por rupturas. En la práctica, las tuberías están sometidas a esfuerzos longitudinales y circunferenciales, esto implica que el esfuerzo longitudinal podría ser Poisson veces el esfuerzo circunferencial, para el caso de presión interna. Si la tubería se encuentra fija y cerrada en los extremos, la relación es 0.5 . Generalmente, la respuesta no lineal es debida a la formación de grietas en la matriz, a mayor carga estas grietas se incrementan. La ISO 14692 es el estándar principal para predecir la presión máxima de servicio para tuberías GRP [18].
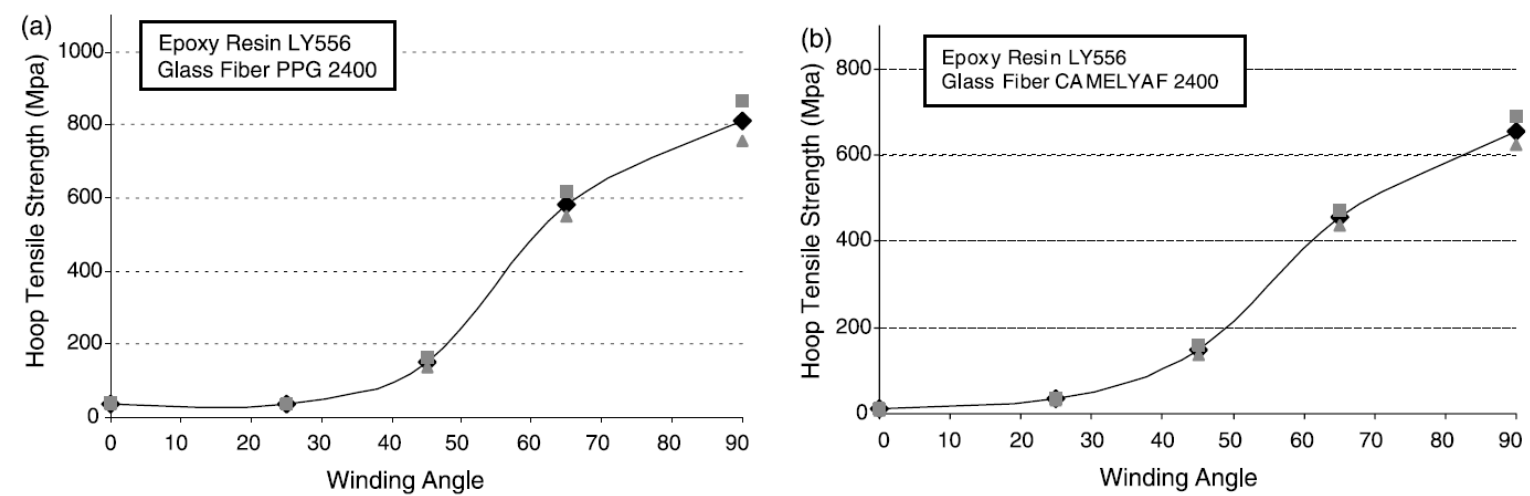

Figura 3. Curvas de esfuerzo vs diferentes ángulos de enrollamiento para tuberías de epoxi-fibra de vidrio o carbono. Fuente: [16]. 


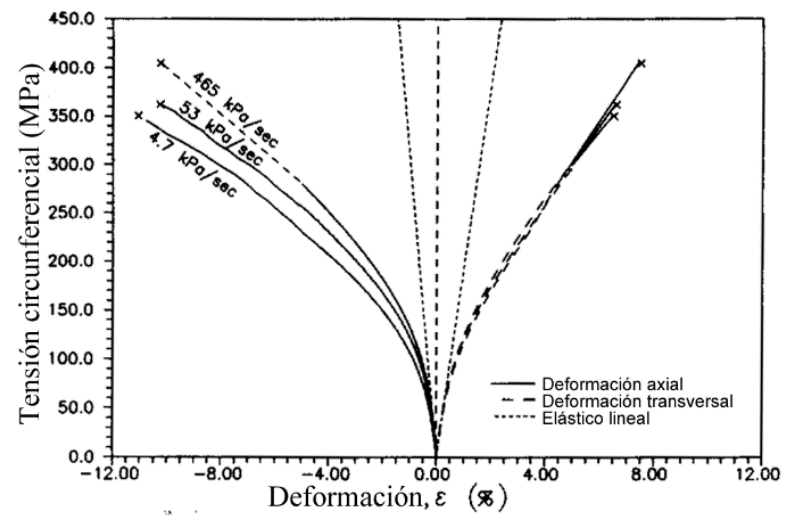

Figura 4. Curvas de esfuerzo deformación en una tubería de fibra de vidrio epoxi con una relación de carga 1 Circunferencial: 0 Axial. Fuente. [17].

\subsection{Laminado}

Un laminado es un conjunto de láminas apiladas unas sobre otras y que, su proceso de fabricación, garantiza la continuidad de la matriz en la dirección ortogonal al plano de las láminas. En otras palabras, cada lámina trabaja conjuntamente con las otras.

Un tipo de apilamiento del laminado que suele utilizarse con mucha frecuencia, y que corresponde al modelo de la tubería Fiberspar, son los denominados laminados simétricos. Para definirlos, es necesario establecer el concepto de plano medio o plano de simetría que separa en dos mitades, del mismo espesor, el laminado. En este plano se consideran los ejes $x$ e $y$, y un eje $z$, ortogonal al plano. Cada una de las láminas se define por sus coordenadas $z_{i}$, de su cara inferior (más próxima al plano medio), y $z_{i+1}$, de su cara superior (más alejada del plano medio) y por la orientación de las fibras en su interior respecto a una dirección de referencia que, usualmente, coincide con el eje $x$ definido anteriormente. Un laminado es simétrico cuando la secuencia de apilamiento de las láminas a uno y otro lado del plano medio son idénticas.

\subsection{Modelo de elasticidad para materiales compuestos}

Consideremos los vectores de tensiones $\boldsymbol{\sigma}=$ $\left\{\sigma_{\mathrm{xx}}, \sigma_{\mathrm{yy}}, \sigma_{\mathrm{xy}}\right\}^{T}$, desplazamientos $\mathbf{u}$ y deformaciones $\boldsymbol{\varepsilon}$, definidos sobre el dominio $\Omega \subset \mathbb{R}^{2}$. Tomemos b como las cargas volumétricas, $\mathbf{t}$ las tracciones de Neumann y $\overline{\mathbf{u}}$ las condiciones de Dirichlet. El problema de elasticidad se define como encontrar $\mathbf{u}$ tal que cumpla:

$$
\begin{gathered}
\mathbf{L}^{\mathrm{T}} \boldsymbol{\sigma}+\mathbf{b}=0 \text { en } \Omega \\
\mathbf{G} \boldsymbol{\sigma}=\mathbf{t} \text { en } \Gamma_{\mathrm{N}} \\
\mathbf{u}=\overline{\mathbf{u}} \text { en } \Gamma_{\mathrm{D}} \\
\boldsymbol{\varepsilon}(\mathbf{u})=\mathbf{L u}
\end{gathered}
$$

$$
\boldsymbol{\sigma}=\mathbf{C} \boldsymbol{\varepsilon}(\mathbf{u}),
$$

donde $\mathbf{L}$ es un operador diferencial, $\mathbf{G}$ es un operador que proyecta las tensiones para el equilibrio en el contorno y C es la matriz con la elasticidad del material.

El problema anterior expresado en su forma variacional se escribe:

Encontrar $\mathbf{u} \in \boldsymbol{V}$ tal que $\forall \mathrm{v} \in \boldsymbol{V}$ :

$$
\begin{array}{r}
\int_{\Omega} \boldsymbol{\varepsilon}(\mathbf{v})^{\mathrm{T}} \mathbf{D} \boldsymbol{\varepsilon}(\mathbf{u}) \mathrm{d} \Omega \\
=\int_{\Omega} \mathbf{v}^{\mathrm{T}} \mathbf{b} \mathrm{d} \Omega \\
+\int_{\Gamma_{\mathrm{N}}} \mathbf{v}^{\mathrm{T}} \mathbf{t} \mathrm{d} \Gamma,
\end{array}
$$

donde $\boldsymbol{V}=\left\{\mathbf{v} \mid \mathbf{v} \in\left[\mathbf{H}^{1}(\boldsymbol{\Omega})\right]^{2}, \mathbf{v}_{\boldsymbol{\Gamma}}=\mathbf{0}\right\}$.

En un material compuesto las fibras pueden estar orientadas en múltiples direcciones arbitrarias. Dependiendo de su disposición, el material presenta comportamientos marcadamente diferenciados en sus respectivas direcciones. Considerando los planos de simetría ortogonales del modelo, el material es de tipo ortótropo. La relación tensión deformación de la ley constitutiva en (5) se puede expresar en términos de la matriz de rigidez $\mathbf{C}$ como:

$$
\left\{\begin{array}{c}
\sigma_{\mathrm{x}} \\
\sigma_{\mathrm{y}} \\
\sigma_{\mathrm{z}} \\
\tau_{\mathrm{yz}} \\
\tau_{\mathrm{xz}} \\
\tau_{\mathrm{xy}}
\end{array}\right\}=
$$

$$
\left[\begin{array}{cccccc}
\mathrm{C}_{11} & \mathrm{C}_{12} & \mathrm{C}_{13} & 0 & 0 & 0 \\
\mathrm{C}_{21} & \mathrm{C}_{22} & \mathrm{C}_{23} & 0 & 0 & 0 \\
\mathrm{C}_{31} & \mathrm{C}_{32} & \mathrm{C}_{33} & 0 & 0 & 0 \\
0 & 0 & 0 & \mathrm{C}_{44} & 0 & 0 \\
0 & 0 & 0 & 0 & \mathrm{C}_{55} & 0 \\
0 & 0 & 0 & 0 & 0 & \mathrm{C}_{66}
\end{array}\right]\left\{\begin{array}{c}
\varepsilon_{\mathrm{x}} \\
\varepsilon_{\mathrm{y}} \\
\varepsilon_{\mathrm{z}} \\
\gamma_{\mathrm{yz}} \\
\gamma_{\mathrm{xz}} \\
\gamma_{\mathrm{xy}}
\end{array}\right\}
$$

donde el comportamiento del material puede ahora definirse mediante 9 constantes independientes. Las constantes $\mathrm{C}_{\mathrm{ij}}$ se obtienen a partir de la teoría clásica de laminados considerando las propiedades elásticas de la fibra y la matriz. Se puede apreciar que las deformaciones angulares y las deformaciones longitudinales están desacopladas de las tensiones normales y las tensiones tangenciales, respectivamente, debido a los términos nulos. Además, no hay interacción entre las tensiones tangenciales y las deformaciones angulares en los diferentes planos.

\subsubsection{Rigidez plana de laminados simétricos}

Los laminados simétricos se caracterizan por tener un plano de simetría, que separa las dos mitades de laminado con el mismo espesor de lámina y orientación de las fibras, tal es el caso de la tubería Fiberspar. La Figura 5 
representa una capa de material compuesto de la tubería Fiberspar, donde se puede observar como están enrolladas las láminas de fibra de vidrio y epoxi, además de la identificación de las coordenadas según la dirección de la fibra en la lámina, donde el ángulo de orientación de las fibras es con respecto al eje axial.

Las propiedades en dirección de la fibra difieren de las propiedades en dirección de los ejes principales del cilindro (axial, radial, circunferencial). Para identificar las propiedades de la tubería es necesario conocer y establecer las relaciones con respecto a las direcciones del material compuesto.

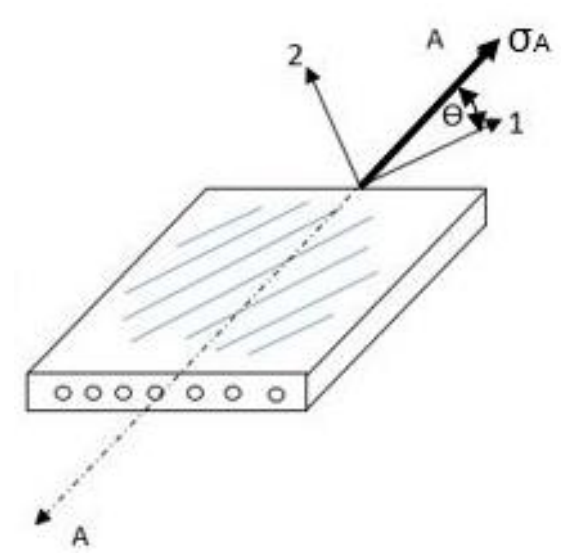

Figura 5. Esquema de la capa de laminado epoxi-fibra de vidrio, donde 1 es la dirección de la fibra y 2 la dirección transversal de la fibra. Fuente. Elaboración propia.

Dadas las características geométricas de la lámina, se asume un estado de tensión plana. La relación deformación tensión para una lámina unidireccional se puede expresar en función de la matriz de flexibilidad $\mathbf{S}=\mathbf{C}^{-1}$ como:

$$
\begin{aligned}
\left\{\begin{array}{c}
\varepsilon_{1} \\
\varepsilon_{2} \\
\gamma_{12}
\end{array}\right\}= & {\left[\begin{array}{ccc}
S_{11} & S_{12} & 0 \\
S_{21} & S_{22} & 0 \\
0 & 0 & S_{66}
\end{array}\right]\left\{\begin{array}{c}
\sigma_{1} \\
\sigma_{2} \\
\tau_{12}
\end{array}\right\} } \\
= & {\left[\begin{array}{ccc}
\frac{1}{E_{1}} & -\frac{v_{21}}{E_{2}} & 0 \\
-\frac{v_{12}}{E_{1}} & \frac{1}{E_{2}} & 0 \\
0 & 0 & \frac{1}{G_{12}}
\end{array}\right]\left\{\begin{array}{c}
\sigma_{1} \\
\sigma_{2} \\
\tau_{12}
\end{array}\right\} }
\end{aligned}
$$

donde las componentes de las matrices de flexibilidad y rigidez se han sustituido por las correspondientes relaciones con las constantes elásticas de la lámina $E_{1}$, $E_{2}, G_{12}, v_{12}$, cuyos valores pueden estimarse a partir de las propiedades de los materiales constituyentes. Las láminas unidireccionales tienen, sin embargo, unas direcciones preferentes asociadas a la orientación de la fibra del refuerzo o a la simetría de los planos. Por ello, es conveniente utilizar dos sistemas de coordenadas: uno para definir los ejes locales $(1,2)$, cuya primera dirección coincide con la dirección de la fibra, y otro para definir los ejes globales $(x, y, z)$.

Cada orientación de lámina utiliza un sistema de coordenadas local, siendo preciso referir la respuesta individual de cada lámina al sistema de coordenadas global o viceversa. Para la transformación de las componentes de tensión y deformación entre los sistemas de coordenadas de ecuaciones globales y los del material compuesto se emplea la matriz de rotación de tensiones:

$$
\begin{aligned}
& \mathrm{T}_{\sigma}(\theta)= \\
& {\left[\begin{array}{ccc}
\cos ^{2} \theta & \operatorname{sen}^{2} \theta & 2 \operatorname{sen} \theta \cos \theta \\
\operatorname{sen}^{2} \theta & \cos ^{2} \theta & -2 \operatorname{sen} \theta \cos \theta \\
-\operatorname{sen} \theta \cos \theta & \operatorname{sen} \theta \cos \theta & \cos ^{2} \theta-\operatorname{sen}^{2} \theta
\end{array}\right]}
\end{aligned}
$$$$
\left\{\begin{array}{c}
\sigma_{1} \\
\sigma_{2} \\
\tau_{12}
\end{array}\right\}=T_{\sigma}(\theta)\left\{\begin{array}{c}
\sigma_{\mathrm{x}} \\
\sigma_{\mathrm{y}} \\
\tau_{\mathrm{xy}}
\end{array}\right\},
$$

Para el caso de las deformaciones se tiene

$$
\begin{aligned}
& \left\{\begin{array}{c}
\varepsilon_{1} \\
\varepsilon_{2} \\
\gamma_{12}
\end{array}\right\}=T_{\varepsilon}(\theta)\left\{\begin{array}{c}
\varepsilon_{\mathrm{x}} \\
\varepsilon_{\mathrm{y}} \\
\gamma_{\mathrm{xy}}
\end{array}\right\}, \\
& T_{\varepsilon}(\theta)= \\
& {\left[\begin{array}{ccc}
\cos ^{2} \theta & \operatorname{sen}^{2} \theta & \operatorname{sen} \theta \cos \theta \\
\operatorname{sen}^{2} \theta & \cos ^{2} \theta & -\operatorname{sen} \theta \cos \theta \\
-2 \operatorname{sen} \theta \cos \theta & 2 \operatorname{sen} \theta \cos \theta & \cos ^{2} \theta-\operatorname{sen}^{2} \theta
\end{array}\right]}
\end{aligned}
$$

Las relaciones tensión deformación conllevan ciertas transformaciones contenidas en la ecuación (11):

$$
\begin{aligned}
& \left\{\begin{array}{c}
\sigma_{\mathrm{x}} \\
\sigma_{\mathrm{y}} \\
\tau_{\mathrm{xy}}
\end{array}\right\}= \\
& \mathrm{T}_{\sigma}^{-1}(\theta)\left[\begin{array}{ccc}
\mathrm{Q}_{11} & \mathrm{Q}_{12} & 0 \\
\mathrm{Q}_{21} & \mathrm{Q}_{22} & 0 \\
0 & 0 & \mathrm{Q}_{66}
\end{array}\right] \mathrm{T}_{\varepsilon}(\theta)\left\{\begin{array}{c}
\varepsilon_{\mathrm{x}} \\
\varepsilon_{\mathrm{y}} \\
\gamma_{\mathrm{xy}}
\end{array}\right\} \\
& =\left[\begin{array}{ccc}
\overline{\mathrm{Q}}_{11} & \overline{\mathrm{Q}}_{12} & \overline{\mathrm{Q}}_{16} \\
\overline{\mathrm{Q}}_{12} & \overline{\mathrm{Q}}_{22} & \overline{\mathrm{Q}}_{26} \\
\overline{\mathrm{Q}}_{16} & \overline{\mathrm{Q}}_{26} & \overline{\mathrm{Q}}_{66}
\end{array}\right]\left\{\begin{array}{c}
\varepsilon_{\mathrm{x}} \\
\varepsilon_{\mathrm{y}} \\
\gamma_{\mathrm{xy}}
\end{array}\right\}
\end{aligned}
$$

Y, por tanto, las relaciones de esfuerzo y deformación en una lámina ortótropa pueden ser escritas como:

$$
\begin{aligned}
& \left\{\begin{array}{c}
\varepsilon_{\mathrm{x}} \\
\varepsilon_{\mathrm{y}} \\
\gamma_{\mathrm{xy}}
\end{array}\right\}= \\
& \mathrm{T}_{\varepsilon}^{-1}(\theta)\left[\begin{array}{ccc}
\mathrm{S}_{11} & \mathrm{~S}_{12} & 0 \\
\mathrm{~S}_{21} & \mathrm{~S}_{22} & 0 \\
0 & 0 & \mathrm{~S}_{66}
\end{array}\right] \mathrm{T}_{\sigma}(\theta)\left\{\begin{array}{c}
\sigma_{\mathrm{x}} \\
\sigma_{\mathrm{y}} \\
\tau_{\mathrm{xy}}
\end{array}\right\} \\
& =\left[\begin{array}{lll}
\overline{\mathrm{S}}_{11} & \overline{\mathrm{S}}_{12} & \overline{\mathrm{S}}_{16} \\
\overline{\mathrm{S}}_{12} & \overline{\mathrm{S}}_{22} & \overline{\mathrm{S}}_{26} \\
\overline{\mathrm{S}}_{16} & \overline{\mathrm{S}}_{26} & \overline{\mathrm{S}}_{66}
\end{array}\right]\left\{\begin{array}{c}
\sigma_{\mathrm{x}} \\
\sigma_{\mathrm{y}} \\
\tau_{\mathrm{xy}}
\end{array}\right\}
\end{aligned}
$$


Siendo las matrices $\overline{\mathrm{Q}}$ y $\overline{\mathrm{S}}$ las denominadas matrices reducidas transformadas (o no orientadas) de rigidez y flexibilidad, respectivamente, referenciadas ahora en ejes globales.

\subsubsection{Solución de elementos finitos.}

Usando una formulación convencional para la discretización de elementos finitos mediante Galerkin en el problema anterior, se busca la solución $\mathbf{u}^{\mathrm{h}} \in \mathbf{V}^{\mathrm{h}}$ tal que $\forall \mathbf{v} \in \mathbf{V}^{\mathrm{h}}$ :

$$
\begin{gathered}
\int_{\Omega} \boldsymbol{\varepsilon}(\mathbf{v})^{\mathrm{T}} \mathbf{D} \boldsymbol{\varepsilon}\left(\mathbf{u}^{\mathrm{h}}\right) \mathrm{d} \Omega=\int_{\Omega} \mathbf{v}^{\mathrm{T}} \mathbf{b} \mathrm{d} \Omega \\
+\int_{\Gamma_{\mathrm{N}}} \mathbf{v}^{\mathrm{T}} \mathbf{t} \mathrm{d} \Gamma
\end{gathered}
$$

la cual se resuelve utilizando la teoría clásica de elementos finitos [19], [20], dando como resultado el sistema lineal $\mathbf{K U}=\mathbf{F}$, siendo $\mathbf{K}$ la matriz de rigidez, $\mathbf{U}$ el vector de desplazamientos, y $\mathbf{F}$ el vector de fuerzas equivalentes.

\subsection{Criterios de falla}

Los materiales compuestos no son homogéneos, son anisótropos y quebradizos. Esto determina los diferentes modos de falla del material, algunos relacionados con la falla de los constituyentes y otros relacionados con la falla de la interfase [21].

En las fibras se pueden considerar dos modos de falla diferentes: relacionado con una carga a tracción, relacionado con una carga a compresión. Una característica de la fibra es que no suele mostrar deformación plástica, estando relacionada la falla con un fenómeno de redistribución de esfuerzos a las fibras vecinas. Esta redistribución puede causar una nueva ruptura de fibras. En el caso de una carga a compresión, el micropandeo progresivo de las fibras tiene lugar hasta que las fibras se rompen.

En la matriz, la microfisuración es el principal modo de falla. Esto equivale a grietas de la matriz paralelas a la dirección de la fibra sobre el espesor completo de la lámina y, especialmente, para aquellas láminas en las que el refuerzo no está en la misma dirección de la carga aplicada.

Otro modo de falla común es la desunión, que equivale a una pérdida de adhesión y un deslizamiento relativo entre la fibra y la matriz, debido a las diferencias en los esfuerzos cortantes de deformación en la interfase fibramatriz [22].

Para el diseño con materiales compuestos se suelen considerar criterios interactivos de falla que tienen en cuenta las interacciones de las tensiones. Entre los criterios interactivos más comunes están Tsai-Hill y Tsai$\mathrm{Wu}[23]$.

\subsubsection{Criterio de Tsai-Hill.}

Este es un criterio basado en los criterios de falla polinomiales y es uno de los criterios más usados y con resultados más ajustados a valores experimentales [21], [23]. La siguiente ecuación representa el criterio de TsaiHill

$$
\left(\frac{\sigma_{1}}{S_{1 u}}\right)^{2}+\left(\frac{\sigma_{2}}{S_{2 u}}\right)^{2}+\left(\frac{\tau_{12}}{S_{12 u}}\right)^{2}-\left(\frac{\sigma_{1} \sigma_{2}}{S_{1 u}^{2}}\right)<1
$$

donde $S_{1 u}$ es la resistencia de la lámina a tracción en dirección longitudinal, $\mathrm{S}_{2 \mathrm{u}}$ en dirección transversal y $\mathrm{S}_{12 \mathrm{u}}$ la resistencia de la lámina por cortadura en el plano. Los subíndices 1, 2 y 12 representan los esfuerzos de tensión paralelos y transversales a la dirección de la lámina compuesta y el esfuerzo cortante, respectivamente, al ser el material transversalmente isótropo. La ecuación indica que la falla no ocurrirá mientras sea menor que uno. La desventaja de Tsai-Hill es que no diferencia entre resistencia a la tensión de fallo y de compresión. Para el caso de carga uniaxial fuera de ejes orientados se tiene:

$$
\begin{gathered}
\frac{1}{\sigma_{\phi}^{2}}>\frac{\cos \varphi^{2}\left(\cos ^{2} \varphi-\operatorname{sen}^{2} \varphi\right)}{S_{1 \mathrm{u}}^{2}}+\frac{\operatorname{sen}^{4} \varphi}{S_{2 \mathrm{u}}^{2}} \\
+\frac{\cos ^{2} \varphi \operatorname{sen}^{2} \varphi}{\mathrm{S}_{12 \mathrm{u}}^{2}}<1
\end{gathered}
$$

donde $\varphi$ representa el ángulo entre las fibras y la dirección de la carga.

\subsubsection{Criterio de Tsai-Wu.}

Basado en la teoría de fallo de la energía total de deformación de Beltrami, el fallo está determinado por la siguiente expresión [21], [23]:

$$
\begin{aligned}
\mathrm{f}_{1} \sigma_{1}+\mathrm{f}_{2} \sigma_{2}+\mathrm{f}_{6} \tau_{12}+\mathrm{f}_{11} \sigma_{1}^{2}+\mathrm{f}_{22} \sigma_{2}^{2} \\
+2 \mathrm{f}_{12} \sigma_{1} \sigma_{2}+\mathrm{f}_{66} \tau_{12}^{2}<1
\end{aligned}
$$

donde $f_{1}, f_{2}, f_{6}, f_{11}, f_{22}, f_{66}$ son descritos en términos de las resistencias últimas en las direcciones principales y $\mathrm{f}_{12}$ es determinado experimentalmente bajo carga de tensión biaxial. Este criterio puede ser aplicado a una lámina para encontrar la primera falla en la capa. TsaiWu es ampliamente usado en el análisis de modelos progresivos de daño para laminados ya que permite determinar los fallos tridimensionales bajo una misma expresión. 


\section{RESULTADOS}

A continuación, se explica el procedimiento experimental y los resultados de simulación numérica para validar los resultados.

\subsection{Resistencia a la tensión aparente}

Se realizó en laboratorio la prueba de resistencia a tracción para obtener valores de la tensión normal aparente bajo la norma ASTM D-2290 [24] utilizando un segmento de anillo partido. La probeta es una muestra de tubería tal como se observa en la Figura 6, el número de probetas ensayadas fueron cinco en total. La norma es aplicable a tuberías compuestas de resina termoestable reforzada con fibras.
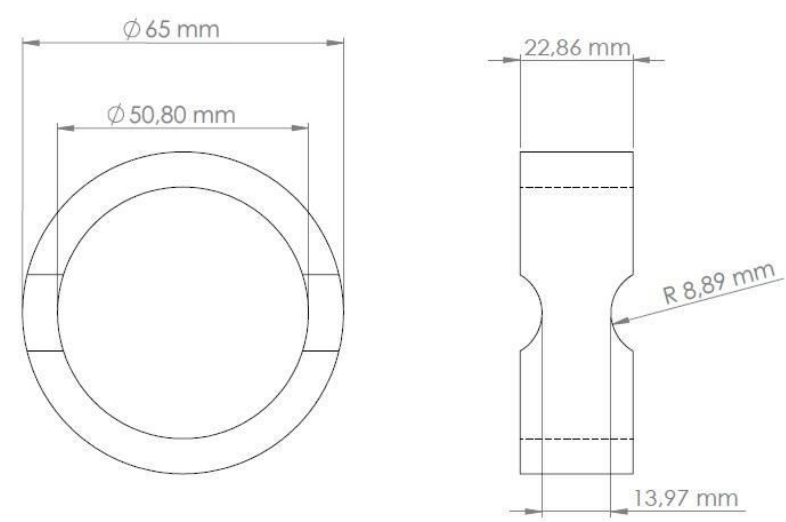

Figura 6. Geometría de la probeta del ensayo ASTM D2290. Fuente. Elaboración propia.

En la Tabla 1 se muestran los valores experimentales de los esfuerzos de tensión aparente que actúa sobre el área mínima de la probeta de ensayo realizados a cinco muestras en total, también es registrada la carga máxima necesaria para la rotura y su desviación estándar s.

Tabla 1. Resultados de las pruebas de laboratorio a cinco muestras de tubería Fiberspar para carga máxima.

\begin{tabular}{|c|c|c|c|}
\hline Muestra & $\begin{array}{c}\text { Carga } \\
\text { máxima } \\
(\mathbf{N})\end{array}$ & $\begin{array}{c}\text { Esfuerzo } \\
\text { último } \\
(\mathbf{M P a})\end{array}$ & $\begin{array}{c}\text { Área } \\
\left(\mathbf{m m}^{\wedge} \mathbf{2}\right)\end{array}$ \\
\hline 1 & 13337 & 75.81 & 88.13 \\
\hline 2 & 15305 & 85.12 & 89.87 \\
\hline 3 & 13046 & 74.15 & 87.96 \\
\hline 4 & 15501 & 78.81 & 98.35 \\
\hline 5 & 16809 & 93.26 & 90.11 \\
\hline Prom. & 14807 & 81.44 & 90.88 \\
\hline $\mathrm{s}$ & 15571 & 7.815 & 4.29 \\
\hline
\end{tabular}

Fuente. Elaboración propia.

\subsection{Modelo numérico}

En esta sección se describe el modelo numérico realizado por elementos finitos. Se define el modelo del material y las diferentes condiciones de contorno utilizadas para los análisis de la integridad estructural de la tubería.

\subsubsection{Dimensiones y modelo del material.}

Para la definición de la geometría del modelo, la Tabla 2 registra las dimensiones de las diferentes capas constitutivas.

Tabla 2. Características geométricas de las capas de la tubería Fiberspar.

\begin{tabular}{ccccccc}
\hline & \multicolumn{2}{c}{$\begin{array}{c}\text { Diámetro } \\
\text { interno }\end{array}$} & \multicolumn{2}{c}{$\begin{array}{c}\text { Diámetro } \\
\text { externo }\end{array}$} & \multicolumn{2}{c}{ Espesor } \\
\cline { 2 - 7 } Capas & (in) & (mm) & (in) & (mm) & (in) & (mm) \\
\hline $\begin{array}{c}\text { Polietileno } \\
\text { interior }\end{array}$ & 2 & 50.8 & 2.196 & 55.78 & 0.09 & 2.3 \\
$\begin{array}{c}\text { Laminado epoxi- } \\
\text { fibra de vidrio } \\
\text { Polietileno } \\
\text { exterior }\end{array}$ & 2.196 & 55.78 & 2.354 & 59.79 & 0.09 & 2.3 \\
\hline
\end{tabular}

Fuente. Elaboración propia.

La capa de refuerzo está compuesta por 4 láminas apiladas con orientaciones de $\pm 55^{\circ}$, las características de la configuración de dicho apilamiento mostrada en la Tabla 3 se puede observar el espesor para cada lámina y espesor total de la capa de refuerzo.

Tabla 3. Características de la capa de laminado epoxifibra de vidrio requeridas para el modelamiento de la tubería Fiberspar.

\begin{tabular}{lcccc}
\hline & $\begin{array}{c}\text { Ángulo de las } \\
\text { fibras por } \\
\text { lamina }\left({ }^{\circ}\right)\end{array}$ & $\begin{array}{c}\text { Espesor por } \\
\text { lámina }\end{array}$ & $\begin{array}{c}\text { Espesor } \\
\text { total }\end{array}$ \\
\hline $\begin{array}{c}\text { Epoxy - Fibra } \\
\text { de vidrio }\end{array}$ & {$[55 /-55 / 55 /-55]$} & 0.02 & 0.502 & 0.079 \\
\hline
\end{tabular}

Fuente. Elaboración propia.

Las propiedades mecánicas de las capas empleadas en la simulación se encuentran en la Tabla 4. Los resultados para los módulos de Young, coeficientes de Poisson y módulos de rigidez a cizalladura para la capa de refuerzo corresponden a los valores calculados a partir de la teoría clásica de laminados, según se indica en las secciones anteriores. En la tabla, el subíndice 1 indica la dirección de la fibra, el subíndice 2, la dirección transversal a la fibra, y el subíndice 3 , hace referencia a la dirección perpendicular al plano 12 . 
La capa de polietileno de alta densidad (HDPE) tiene comportamiento isótropo, sus propiedades son las mismas en todas las direcciones, así que las propiedades en la dirección 1 son las mismas para todas las direcciones. EL HDPE se caracteriza por tener una alta capacidad de resistencia térmica y también resistente al impacto. El refuerzo epoxi-fibra de vidrio es un material ortótropo, por eso sus propiedades cambian en las direcciones principales, tal como se observa en la Tabla 4. La capa de refuerzo está diseñada para soportar los altos esfuerzos y presiones generados en la tubería. Estos materiales fueron consultados e identificados de acuerdo a la patente de la tubería y catálogos de fabricantes [15], [25].

\subsubsection{Mallado}

Con el fin de controlar el error de discretización, se realizaron pruebas de independencia de malla para verificar la convergencia de la solución al rango asintótico. Los resultados se observan en la Figura 7 y en la Tabla 5. El refinamiento se realizó para la capa de refuerzo, hasta obtener una variación del esfuerzo circunferencial menor al $1 \%$. El mallado se realiza con elementos hexaédricos lineales para la capa de epoxifibra de vidrio y para las capas interior y exterior de polietileno. Entre las capas se definieron condiciones de contacto lineales tipo penalti.

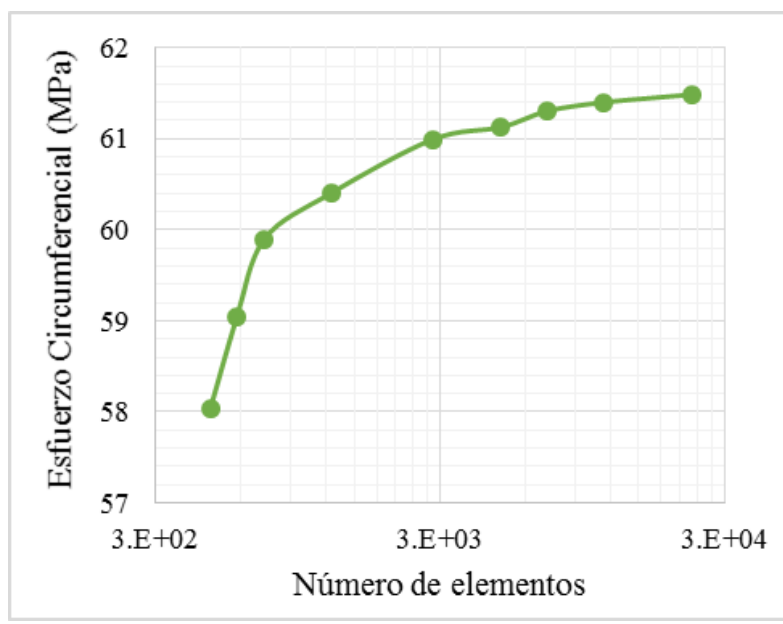

Figura 7. Convergencia de los esfuerzos sobre la capa epoxi-fibra de vidrio. Fuente. Elaboración propia.

Tabla 4. Propiedades físicas de cada capa de la tubería Fiberspar.

\begin{tabular}{|c|c|c|c|c|c|c|c|c|c|c|}
\hline \multirow{2}{*}{ Capa } & \multicolumn{3}{|c|}{ Módulo de Young } & \multicolumn{3}{|c|}{$\begin{array}{c}\text { Coeficiente de } \\
\text { Poisson }\end{array}$} & \multicolumn{3}{|c|}{$\begin{array}{l}\text { Módulo de } \\
\text { cizalladura }\end{array}$} & \multirow{2}{*}{$\begin{array}{r}\text { Densidad } \\
\left(\mathrm{kg} / \mathrm{m}^{\wedge} 3\right)\end{array}$} \\
\hline & $\begin{array}{c}\mathrm{E}_{1} \\
(\mathrm{MPa})\end{array}$ & $\begin{array}{c}\mathrm{E}_{2} \\
(\mathrm{MPa})\end{array}$ & $\begin{array}{c}\mathrm{E}_{3} \\
(\mathrm{MPa})\end{array}$ & $\mathrm{v}_{12}$ & $v_{23}$ & $v_{13}$ & $\begin{array}{c}\mathrm{G}_{12} \\
(\mathrm{MPa})\end{array}$ & $\begin{array}{c}\mathrm{G}_{23} \\
(\mathrm{MPa})\end{array}$ & $\begin{array}{c}\mathrm{G}_{13} \\
(\mathrm{MPa})\end{array}$ & \\
\hline Polietileno interior & 1340 & I & I & 0.4 & I & I & 478.5 & I & I & 970 \\
\hline $\begin{array}{c}\text { Laminado epoxi-fibra } \\
\text { de vidrio }\end{array}$ & 35000 & 9000 & 9000 & 0.28 & 0.4 & 0.28 & 4700 & 3500 & 4700 & 1850 \\
\hline Polietileno exterior & 1340 & / & I & 0.4 & I & I & 478.5 & I & I & 970 \\
\hline
\end{tabular}

Fuente. Elaboración propia. 
Tabla 5. Convergencia de los esfuerzos circunferenciales máximos del laminado.

\begin{tabular}{ccc}
\hline $\begin{array}{c}\text { Número de } \\
\text { elementos }\end{array}$ & $\begin{array}{c}\text { Esfuerzos } \\
\text { Max (MPa) }\end{array}$ & Error \\
\hline 468 & 58.034 & $1.723 \%$ \\
580 & 59.034 & $1.467 \%$ \\
726 & 59.9 & $0.841 \%$ \\
1247 & 60.404 & $0.977 \%$ \\
2837 & 60.994 & $0.220 \%$ \\
4930 & 61.128 & $0.288 \%$ \\
7140 & 61.304 & $0.157 \%$ \\
11264 & 61.4 & $0.140 \%$ \\
23145 & 61.486 & \\
\hline
\end{tabular}

Fuente. Elaboración propia.

La Figura 8 muestra el mallado final obtenido para el ensamble con una longitud de $10 \mathrm{in}$. El mallado final total de la tubería contiene 74112 elementos y 241215 nodos.

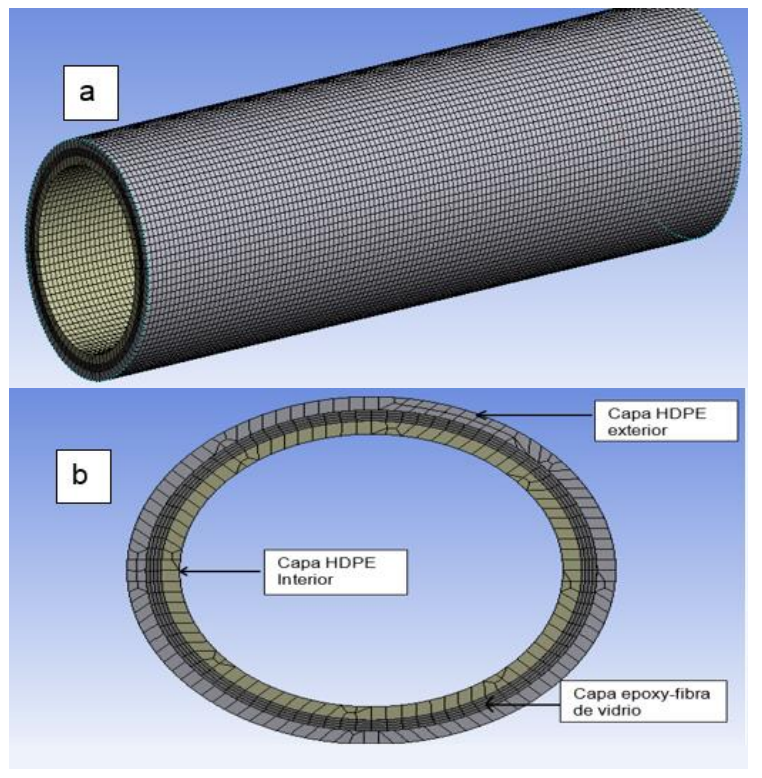

Figura 8. Modelo de EF: (a) Isométrico y (b) vista frontal. Fuente. Elaboración propia.

\subsubsection{Norma ASTM D2290}

Para la simulación de la norma se tuvo en cuenta la carga promedio que actúa sobre el área mínima utilizada en la prueba experimental (ver Tabla 1) correspondiente a un valor de $14807 \mathrm{~N}$. Se modela únicamente media tubería considerando las condiciones de simetría.

La Figura 9 muestra los resultados de la tensión circunferencial presentados sobre la tubería, que para la sección transversal considerada coincide con la tensión normal, con un valor máximo de $346 \mathrm{MPa}$. El esfuerzo aparente calculado sobre el área mínima de la muestra corresponde a $82.312 \mathrm{MPa}$ (ver Tabla 6). Teniendo en cuenta los resultados de laboratorio para la tensión aparente promedio de $81.442 \mathrm{MPa}$, se puede obtener el porcentaje de error relativo que existe entre la prueba experimental y el modelo numérico con un valor igual a $1.06 \%$. Dichos resultados permiten validar el modelo del material y las condiciones de contorno consideradas en el modelo numérico para la tubería Fiberspar.

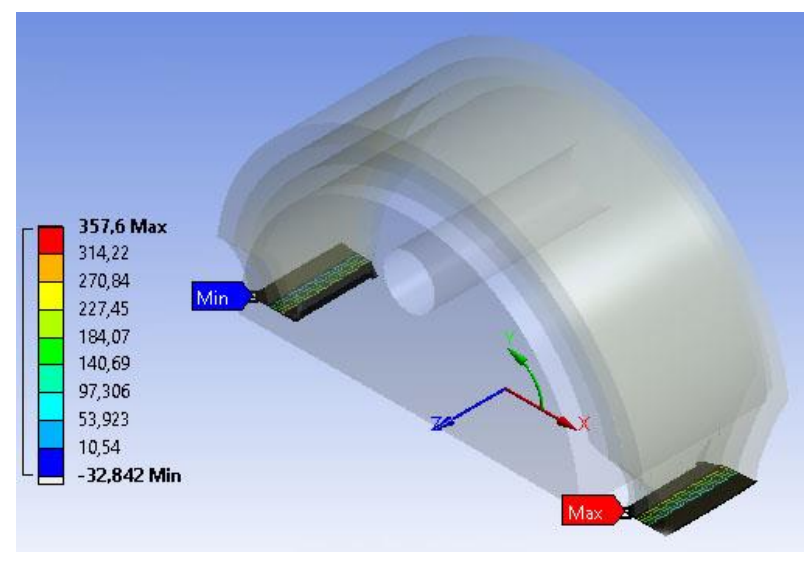

Figura 9. Distribución de tensión normal en la zona de concentración de esfuerzos. Fuente. Elaboración propia.

Tabla 6. Comparación del resultado experimental y de la simulación.

\begin{tabular}{cccc}
\hline \multirow{2}{*}{$\begin{array}{c}\text { Área } \\
\left(\mathbf{m m}^{\wedge} \mathbf{2}\right)\end{array}$} & $\begin{array}{c}\text { Carga } \\
\text { máxima } \\
\text { promedio }(\mathbf{N})\end{array}$ & \multicolumn{2}{c}{ Tensión última (MPa) } \\
\cline { 3 - 4 } & Experimental & Numérico \\
\hline 90.88 & 14807 & 81.442 & 82.312 \\
\hline
\end{tabular}

Fuente. Elaboración propia.

\subsubsection{Condiciones de contorno a la presión de trabajo nominal.}

Las condiciones de contorno que se utilizan son las condiciones de presión nominal o de trabajo de la tubería. Se aplica una presión nominal de 5.17 MPa en dirección normal a la superficie interior. Se restringe el desplazamiento en dirección axial en ambas caras laterales. Este tipo de condición, conocida como abiertaabierta, representa la simetría de una sección de tubo y afecta el valor de la tensión longitudinal calculada. Condiciones distintas se utilizan para simular codos y cierre por válvulas en la línea. 
3.2.5. Esfuerzos a la presión de trabajo nominal.

Para la presión nominal de trabajo de $5.17 \mathrm{MPa}$ (749 psi), los esfuerzos máximos principales obtenidos se presentan sobre la capa de refuerzo fibra de vidrio, generando un esfuerzo máximo circunferencial de 61.4 MPa o 8917 psi. Esto implica que cuando la tubería está sometida a tensión las capas interior y exterior tienden a apretar la capa de refuerzo, los esfuerzos máximos son los esfuerzos tangenciales. Los esfuerzos tangenciales en la capa exterior de HDPE fueron mayores a los de la capa interior con $4 \mathrm{MPa}$. Los resultados de los análisis respecto a los esfuerzos máximos axiales fueron de 19.9 $\mathrm{MPa}$, mientras que los esfuerzos axiales en las capas interior y exterior fueron de $1.15 \mathrm{MPa}$ y $1.5 \mathrm{MPa}$, respectivamente. Los resultados pueden observarse en la Tabla 7.

El análisis de deformación refleja que la capa que registra mayor deformación es la interior de polietileno con un valor de deformación a lo largo del aro de $0.018 \mathrm{~mm} / \mathrm{mm}$ y un valor mínimo en la capa exterior de $0.01 \mathrm{~mm} / \mathrm{mm}$.

Los resultados indican que la lámina de compuesto de fibra de vidrio más cercana a la capa interior presenta mayor esfuerzo y este va disminuyendo en forma lineal hacia la última capa. En la Figura 10 se representan los esfuerzos longitudinales y circunferenciales generados a lo largo del espesor de la tubería, reflejando como la solicitación es asumida mayormente por la capa de laminado. Las capas de polietileno funcionan para proteger la capa de laminado de la corrosión y trasmitir los mayores esfuerzos al laminado compuesto. Como es de esperarse, los esfuerzos más significativos son los esfuerzos circunferenciales.

Tabla 7. Esfuerzos registrados sobre la tubería Fiberspar a la presión de operación.

\begin{tabular}{|c|c|c|c|c|}
\hline \multirow{2}{*}{ Capa } & \multicolumn{2}{|c|}{$\begin{array}{c}\text { Esfuerzo } \\
\text { circunferencial }\end{array}$} & \multicolumn{2}{|c|}{$\begin{array}{c}\text { Esfuerzo } \\
\text { longitudinal }\end{array}$} \\
\hline & (MPa) & (psi) & (MPa) & (psi) \\
\hline Polietileno interior & 2.1 & 306.1 & 1.15 & 168 \\
\hline $\begin{array}{l}\text { Laminado epoxi- } \\
\text { fibra de vidrio }\end{array}$ & 61.4 & 8917 & 19.9 & 2892 \\
\hline Polietileno exterior & 3.9 & 575 & 1.5 & 223 \\
\hline
\end{tabular}

Fuente. Elaboración propia.

Para realizar una comparación teórica de los resultados obtenidos se utilizó el software Heliux Composite ${ }^{\circledR}$ que proporciona características del comportamiento del material compuesto, basado en una formulación analítica. Los esfuerzos circunferenciales y axiales se ilustran en la Figura 11 y Figura 12.

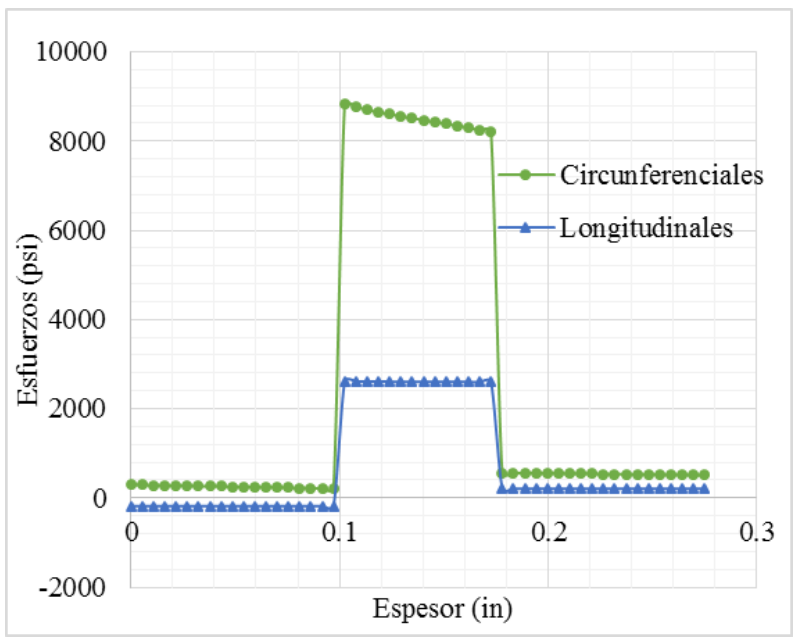

Figura 10. Esfuerzos circunferenciales y axiales sobre la tubería en función del espesor para una presión de 749.8 pis (5.17 MPa). Fuente. Elaboración propia.

Los esfuerzos circunferenciales registrados máximos oscilan entre $8910 \mathrm{psi}(61.4 \mathrm{MPa})$ y $8200 \mathrm{psi}(56 \mathrm{MPa})$, valores muy cercanos a los obtenidos de 8917 psi y 8203 psi (Figura 10). La distribución de tensiones entre capas, a lo largo del espesor, también muestra el mismo comportamiento.

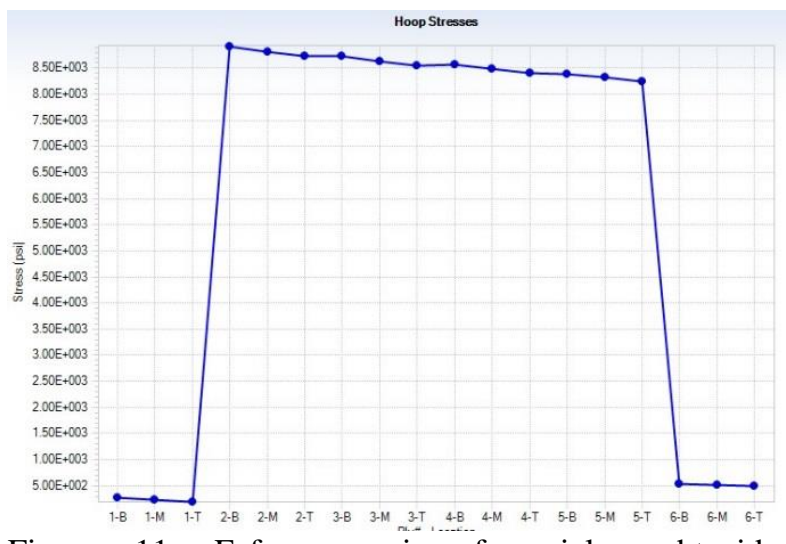

Figura 11. Esfuerzos circunferenciales obtenidos mediante el software Heliux con un valor máximo de 61.4 MPa (8910 psi). Fuente. Elaboración propia.

Para los esfuerzos longitudinales, la Figura 12 muestra que los esfuerzos máximos registrados son 602 psi (4.15 $\mathrm{MPa}$ ), diferentes a los de la simulación de 2600 psi. La diferencia se debe a que Heliux no permite establecer las restricciones de desplazamiento longitudinal en comparación con la simulación. 


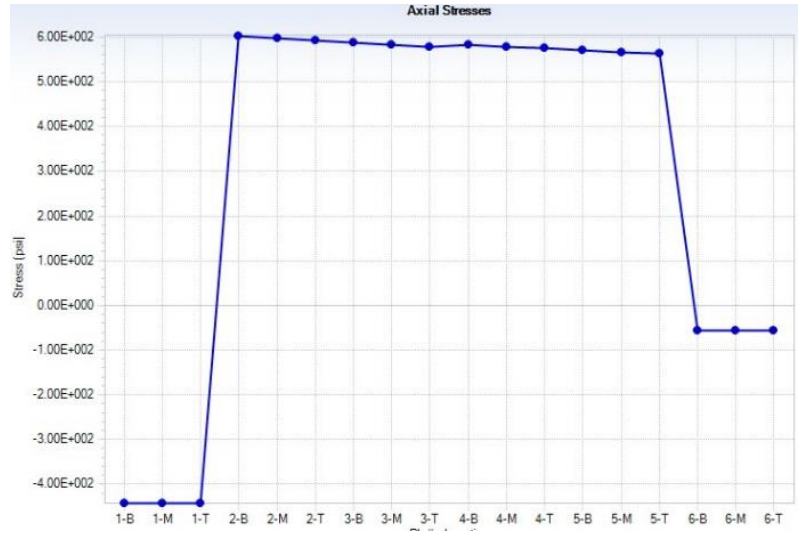

Figura 12. Esfuerzos longitudinales obtenidos mediante el software Heliux con un valor máximo de 4.5 MPa (602 psi). Fuente. Elaboración propia.

3.2.6. Evaluación de criterios de Tsai-Hill y Tsai-Wu. Se tuvieron en cuenta los criterios de fallo de Tsai-Hill y Tsai-Wu para la tubería de material compuesto Fiberspar. Para esto, se emplearon 3 presiones, la primera presión nominal o de trabajo de $5.17 \mathrm{MPa}$, la segunda presión fue la de estallido o explosión de 27.1 MPa y para la tercera presión se consideró una presión intermedia para observar el cambio de criticidad de las fibras de material compuesto. A continuación, se muestran los criterios de fallo en las tres distintas presiones mencionadas anteriormente.

Tabla 8. Resultados de los análisis de criterios de fallo Tsai-Hill Tsai -Wu.

\begin{tabular}{|c|c|c|c|c|}
\hline \multirow{2}{*}{$\begin{array}{c}\text { Presión } \\
\text { (MPa) }\end{array}$} & \multicolumn{2}{|c|}{ Tsail-Hill } & \multicolumn{2}{c|}{ Tsai-Wu } \\
\cline { 2 - 5 } & Max & $\begin{array}{c}\text { Lámina } \\
\text { crítica }\end{array}$ & Max & $\begin{array}{c}\text { Lámina } \\
\text { crítica }\end{array}$ \\
\hline 5.17 & 0.518 & Ninguna & 0.48 & Ninguna \\
\hline 12.5 & 1.25 & Primera & 1.16 & Primera \\
\hline 27 & 2.5 & Todas & 2.7 & Todas \\
\hline
\end{tabular}

Fuente. Elaboración propia.

Utilizando el criterio de Tsai-Hill y Tsai-Wu se confirma que para una presión de trabajo de 5.17 MPa no existe ningún rompimiento de fibra del material, por tanto, no hay fallo, debido a que los criterios de fallo son menores a 1 según los resultados registrados en la Tabla 8. Esta tabla indica que para una presión de $12.5 \mathrm{MPa}$, las fibras registran mayor sobrecarga y la primera lámina interior es la más crítica, puede haber ruptura de fibra mas no un fallo completo de la tubería. Mediante el análisis realizado para una presión de $27 \mathrm{MPa}$ se confirma que hay fallos y todas las capas han superado el valor del criterio por encima de 1 .

\section{CONCLUSIONES}

Se definió numéricamente el modelo del material compuesto para la tubería Fiberspar. Se prestó especial interés en la definición de la capa reforzada con fibras, y se validó el modelo mediante pruebas experimentales. Se recopilaron datos de condiciones de operación en la región para las tuberías Fiberspar.

El error entre los resultados de la prueba de laboratorio según la norma ASTM D2290 y la aproximación numérica es mínimo. Esto valida los parámetros utilizados para definir el modelo computacional del material.

Se puede observar que, cuando la tubería ortótropa está sometida a una presión mayor de $12 \mathrm{MPa}$, la primera lámina de epoxi-fibra de vidrio falla según los criterios de Tsai-Hill y Tsai-Wu, pero esto no quiere decir que la tubería falle por completo. A la presión de estallido de 27.17 MPa la tubería falla en todas las láminas.

Se determinaron los valores de los esfuerzos sobre la tubería cuando esta trabaja a su presión nominal de 5.17 MPa. También se comprobó que la capa de laminado compuesto es la que soporta los mayores esfuerzos con un valor de $62 \mathrm{MPa}$ para los esfuerzos circunferenciales y de 19 MPa para los esfuerzos longitudinales.

En este trabajo, se presentó un modelo numérico para el análisis de la respuesta mecánica de tuberías de material compuesto tipo Fiberspar, de manera que se pueda estimar el efecto de las solicitaciones sobre la línea de transporte y proyectar su uso para condiciones óptimas de servicio. Trabajos futuros incluyen el estudio de fenómenos de fatiga y diferentes modelos de daño para materiales compuestos, aplicables a las tuberías para el transporte de hidrocarburos en la región.

\section{AGRADECIMIENTOS}

Los autores agradecen el apoyo a través del proyecto Capital Semilla - 1742 de la UIS, y del Convenio Marco UIS - ECP No 5222395.

\section{REFERENCIAS}

[1] R. Rafiee, "On the mechanical performance of glass-fibre-reinforced thermosetting-resin pipes: A review," Compos. Struct., vol. 143, pp. 151-164, 2016.

[2] N. Ji, H. Geun, and J. Heum, "Structural analysis and optimum design of GRP pipes based on properties of materials," Constr. Build. Mater., vol. 38, pp. 316-326, 2013.

[3] D. Hull, "Research on composite materials at Liverpool University. I. Failure of filament wound tubes," Phys. Technol., vol. 13, no. 5, 1982. 
[4] J. R. M. de Sousa, G. B. Ellwanger, and E. C. P. Lima, "Modelo tridimensional de elementos finitos para el análisis de esfuerzos de tubos flexibles," Boletín Técnico, vol. 42, no. 2, pp. 1-20.

[5] X. Anping, S. Peng, Z. Jingjing, and Q. Yunxia, "FEA-based Comparison of Two Kinds of Steel Wire Reinforced Composite Pipes," 4th Int. Conf. Intell. Networks Intell. Syst., pp. 184-187, 2011.

[6] K. Yu, E. V. Morozov, M. A. Ashraf, and K. Shankar, "Analysis of flexural behaviour of reinforced thermoplastic pipes considering material nonlinearity," Compos. Struct., vol. 119, pp. 385-393, 2014.

[7] K. Yu, E. V. Morozov, M. a. Ashraf, and K. Shankar, "Numerical analysis of the mechanical behaviour of reinforced thermoplastic pipes under combined external pressure and bending," Compos. Struct., vol. 131, pp. 453-461, 2015.

[8] L. A. Quintero Ortiz, J. R. Arciniegas Villamizar, M. C. Jiménez Romero, and A. Y. Vallen Vargas, "Efecto en las propiedades mecánicas de daños superficiales generados en tubería compuesta flexible para transporte de hidrocarburos.," Rev. Ing., no. 42, pp. 39-48, 2015.

[9] Y. Bai, J. Tang, W. Xu, Y. Cao, and R. Wang, "Collapse of reinforced thermoplastic pipe (RTP) under combined external pressure and bending moment," Ocean Eng., vol. 94, pp. 10-18, 2015.

[10] Y. Reutov, "The calculation of multilayer polymer pipes using finite elements and their application to Gas and Oil pipelines," in 7th International Forum on Strategic Technology (IFOST), 2012, pp. 1-3.

[11] J. L. C. G. de Kanter and J. Leijten, "Thermoplastic Composite Pipe: Analysis and Testing of a Novel Pipe System for Oil \& Gas," in Proceedings of the 17th ICCM, 2009, pp. 1-10.

[12] H. Bakaiyan, H. Hosseini, and E. Ameri, "Analysis of multi-layered filament-wound composite pipes under combined internal pressure and thermomechanical loading with thermal variations," Compos. Struct., vol. 88, no. 4, pp. 532-541, 2009.

[13] M. Xia, H. Takayanagi, and K. Kemmochi, "Bending behavior of filament-wound fiber-reinforced sandwich pipes," Compos. Struct., vol. 56, no. 2, pp. 201-210, 2002.

[14] P. D. Soden, M. J. Hinton, and A. S. Kaddour, "Biaxial test results for strength and deformation of a range of E-glass and carbon fibre reinforced composite laminates. Failure exercise benchmark data," Fail. Criteria Fibre-Reinforced-Polymer Compos., vol. 62, pp. 52-96, 2004.

[15] P. A. Quigley, S. C. Nolet, and J. G. Williams, "Composite spoolable tube," U.S. Patent 6,016,845, 2000.

[16] C. Kaynak, E. S. Erdiller, L. Parnas, and F. Senel, "Use of split-disk tests for the process parameters of filament wound epoxy composite tubes," Polym. Test., vol. 24, no. 5, pp. 648-655, 2005.
[17] M. Carroll, F. Ellyin, D. Kujawski, and A. S. Chiu, "The rate-dependent behaviour of $\pm 55^{\circ}$ filament-wound glass-fibre/epoxy tubes under biaxial loading," Compos. Sci. Technol., vol. 55, no. 95, pp. 391-403, 1995.

[18] A. Majid and M. S. Bin, "Behaviour of composite pipes under multi-axial stress," Newcastle University, 2011.

[19] E. J. Barbero, Finite Element Analysis of Composite Materials Using ANSYS, Second. Boca Raton, U.S.A.: CRC Press, 2013.

[20] O. C. Zienkiewicz, R. L. Taylor, and J. Z. Zhu, The Finite Element Method: its Basis and Fundamentals, 7th ed. 2013.

[21] M. Molinier, "Análisis de los criterios de falla aplicados a los laminados compuestos," Universidad de Buenos Aires, 2005.

[22] L. Arias Maya and L. Vanegas Useche, "Falla de los materiales compuestos laminados," Sci. Tech., no. 25, pp. 113-118, 2004.

[23] M. E. Tuttle, Structural Analysis of Polymeric Composite Materials. New York, U.S.A., 2004.

[24] ASTM Standard, "ASTM 2290-12 Standard Test Method for Apparent Hoop Tensile Strength of Plastic or Reinforced," ASTM B. Stand., pp. 1-8, 2016.

[25] Fiberspar, "Full Reel Program. Certified Installer Training," 2010. 